\section{MYOBLAST TRANSPLANTATION IN THE PORCINE MODEL: A POTENTIAL TECHNIQUE FOR MYOCARDIAL REPAIR}

The use of transgenic cells transplanted in syngeneic rodents has shown modest success, but allogeneic and xenogeneic transplants have not been uniformly successful. To assess the feasibility of xenogeneic and allogeneic myoblast transplantation, we subjected seven adult swine to transplantation of murine atrial tumor cells (xenogeneic), neonatal porcine myocytes (allogeneic), and human fetal cardiomyocytes into the left ventricular wall. After general anesthesia, isolated cells were injected along the anterior and posterior walls of the porcine left ventricle. All the animals were immunosuppressed and observed for 1 month after injection, at which time they were killed and analyzed. This report will present results primarily concerned with the success of human cell transfers. In all injected sites examined, the transplanted cells thrived within the host myocardium with no significant rejection. Transplant cells formed close associations with host myocytes that resembled nascent intercalated disks on electron microscopy. These cells also contained myofibrils and other cell architecture resembling the transplanted cell lines. Additionally, these cells appeared to produce an angiogenic influence resulting in the proliferation of the surrounding microvasculature. We believe that these findings indicate successful xenogeneic and allogeneic myoblast cell transplantation in a large animal model. These experiments set the stage for future studies to assess the ability of these cells to form a syncytium, contract, and potentially repair failed myocardium. (J THORAC CARDIOVASC SURG 1995; 110:1442-8)

Clifford H. Van Meter, Jr., MD (by invitation), William C. Claycomb, PhD (by invitation), Joseph B. Delcarpio, PhD (by invitation),

Duane M. Smith, PhD (by invitation), Helen deGruiter, RN (by invitation), Frank Smart, MD (by invitation), and John L. Ochsner, MD, New Orleans, La.
T he relative shortage of donor hearts continues to fuel the efforts to establish other methods to manage heart failure. Many have expanded the criteria for acceptability of donor hearts and continue to experiment clinically with ventricular assist devices, skeletal cardiomyoplasty, and even total artificial hearts. Simultaneously, research has continued in the arena of cellular transplantation.

Parallel research along this line, with satellite cells

From the Ochsner Medical Institutions and Louisiana State University School of Medicine, New Orleans, La.

Read at the Seventy-fifth Annual Meeting of The American Association for Thoracic Surgery, Boston, Mass., April 23-26, 1995.

Address for reprints: Clifford H. Van Meter, Jr., MD, Ochsner Medical Institutions, 1514 Jefferson Highway, New Orleans, LA 70121.

Copyright (C) 1995 by Mosby-Year Book, Inc.

$0022-5223 / 95 \$ 5.00+0 \quad \mathbf{1 2 / 6 / 6 7 8 6 4}$ in skeletal muscle, has been previously reported. ${ }^{1}$ These cells do not fuse with others or synthesize contractile proteins and are left wedged between the plasma and base membranes of the muscle fiber. They play a primary role in the regeneration of skeletal muscle after injury.

Unlike skeletal muscle, cardiac muscle differentiation is unusual in that it occurs early in fetal life in the dividing cell. The reason is that the heart is one of the first organs to form during development and, as such, gains function while it is still growing by cell division. Because cardiac muscle cells continue to divide as they are differentiating, ${ }^{2}$ it may be that they have two separate developmental programs for terminal differentiation. Ongoing research is attempting to identify the genes that control cardiac myocyte differentiation and the myocyte cell cycle. This report summarizes the results of our pilot studies with experimental cardiomyoblast transplantation. 
Volume 110, Number 5

\section{Methods}

Preparation of adult rat ventricular cardiomyocytes. Ventricular cardiomyocytes were isolated from adult female rats $(200$ to $300 \mathrm{gm}$ ) by means of the protocols of Claycomb and Palazzo ${ }^{3}$ and modified after Claycomb and Lanson. ${ }^{4}$ Rats were anesthetized, and their beating hearts were removed and placed into cold Joklik's medium. Hearts were then attached to a short Friedrick's condenser by a three-way stopcock and perfused retrogradely through the aorta with enzyme-free Joklik's medium. This was followed immediately by retrograde perfusion with Joklik's medium containing type II collagenase in a 1 $\mathrm{mg} / \mathrm{ml}$ concentration. All solutions were maintained at $37^{\circ} \mathrm{C}$ and gassed with a $0.8 \mathrm{~mm}$ filtered mixture of $95 \%$ oxygen and $5 \%$ carbon dioxide. Once softened, the hearts were removed, minced, and subjected to gentle digestion in fresh collagenase by stirring a heated rotating shaker bath. Every 10 minutes, cells were collected, washed in serum-free Joklik's medium, and pooled.

Preparation of AT-1 cells. AT-1 cells were isolated from subcutaneous atrial tumors by means of techniques previously published..$^{5,6}$ The tumors were excised from their subcutaneous location and rinsed in phosphatebuffered saline solution. The tumors were minced in phosphate-buffered saline solution, transferred to a trypsinizing flask containing $0.125 \%$ trypsin, and treated as described earlier for rat ventricles. Cells were counted and pooled.

Preparation of isolated fetal human cardiomyocytes. Fetal human hearts (14 to 18 weeks of gestation) were obtained from Advanced Bioscience Resources, Inc. (Almeda, Calif.). The hearts were procured immediately after abortions, rinsed in saline solution, placed in icecold, sterile, high-glucose Dulbecco's modified Eagle's medium, packed in wet ice, and shipped by overnight carrier. Ventricular and atrial heart muscle cells were isolated by the same procedures and with the same apparatus used previously to isolate cardiac myocytes from adult rats and monkeys. ${ }^{7-10}$ These procedures are easily adaptable to fetal human hearts because at this developmental age they are approximately the same size as hearts from adult rats and squirrel monkeys. Under aseptic conditions, the hearts were perfused in a retrograde manner via the aorta with $1 \mathrm{mg}$ of collagenase per milliliter of Joklik's medium followed by sequential digestion of minced pieces of ventricular or atrial tissue in fresh enzyme solution. Dissociated cells were washed several times in Joklik's medium for purification and injected within several hours after isolation.

Injection of cardiomyocytes and cardiomyblasts into test animals. Seven adult swine were anesthetized with ketamine and barbiturates, intubated, and their lungs ventilated with isoflurane via a pressure-controlled respirator. Venous access was accomplished with a Port-ACath catheter (Pharmacia Biosystems, Piscataway, N.J.) and bretylium was injected as a bolus at a rate of $5 \mathrm{mg} / \mathrm{kg}$ followed by a constant infusion to prevent ventricular arrhythmias.

Using aseptic technique, we made a midline sternotomy and created a pericardial well. We then injected $10 \mu \mathrm{l}$ of cells in solution (approximately $10^{4}$ cardiomyoblasts in Joklik's medium) into the ventricular wall using a 26 - gauge micropuncture needle connected to a tuberculin syringe. Controlled injections were performed with $10 \mathrm{ml}$ of Joklik's medium only. The midline sternotomy was closed and the pericardial and thoracic spaces were evacuated of residual air.

All animals were given cyclosporine (Sandimune, Sandoz Pharmaceuticals, E. Hannover, N.J.) at a rate of 15 $\mathrm{mg} / \mathrm{kg}$ twice a day by intravenous bolus to maintain therapeutic cyclosporine levels. Animals were killed 1 month after injection and their hearts were examined by light and transmission electron microscopes.

One month after cellular implantation, all animals were killed and the hearts excised and prepared for examination by light and electron microscopy as follows.

\section{Microscopy}

Microscopic examination of whole tissue. Isolated hearts were perfused through the coronary tree with a phosphate-buffered $4 \%$ paraformaldehyde- $1 \%$ glutaraldehyde fixative. Excised whole hearts were fixed an additional 7 days in $4.0 \%$ glutaraldehyde and $0.1 \mathrm{~mol} / \mathrm{L}$ sodium cacodylate. Injection sites were located by identifying Prolene sutures (Ethicon, Inc., Somerville, N.J.) implanted at the initial operation. Excised areas were cut into $1 \mathrm{~cm}$ squares and then rinsed for an additional week in $0.1 \mathrm{~mol} / \mathrm{L}$ sodium cacodylate buffer with frequent changes of buffer. Blocks were further sectioned into smaller samples for examination with both electron and light microscopes.

Electron microscopy. Tissue samples were postfixed in $1.0 \%$ osmium tetroxide and $0.1 \mathrm{~mol} / \mathrm{L}$ sodium cacodylate, stained en bloc with $0.5 \%$ aqueous uranyl acetate, dehydrated in acetone, and infiltrated and embedded in Polybed 812 fixative (Polysciences, Inc., Warrington, Pa.). Thin sections were examined with a JEOL 1210 transmission electron microscope (JEOL USA, Inc., Peabody, Mass.), at $60 \mathrm{kV}$. Images were obtained on Kodak 4489 EM film (Eastman Kodak Company, Rochester, N.Y.).

Standard light histologic studies. Tissue blocks were prepared for light microscopy, embedded in paraffin, sectioned, and stained with hematoxylin and eosin. Sections were examined with a Leitz Dialux 20 microscope (Wild Microscopes, Div. of Leica, Inc., Rockleigh, N.J.). Images were captured on Kodak T-Max 100 film at various levels of magnification.

All animals received humane care in compliance with the "Principles of Laboratory Animal Care" formulated by the National Society for Medical Research and the "Guide for the Care and Use of Laboratory Animals" prepared by the Institute of Laboratory Animal Resources and published by The National Institutes of Health (NIH Publication \#86-23, revised 1985) and in conjunction with the Ochsner Medical Institutions Research and Education Division Animal Care Committee.

\section{Results}

All seven laboratory animals received injections of cells without perioperative complication and survived without morbidity until being put to death for harvesting. There was no increase in creatine kinase MB isoenzyme fraction within 24 hours after injec- 


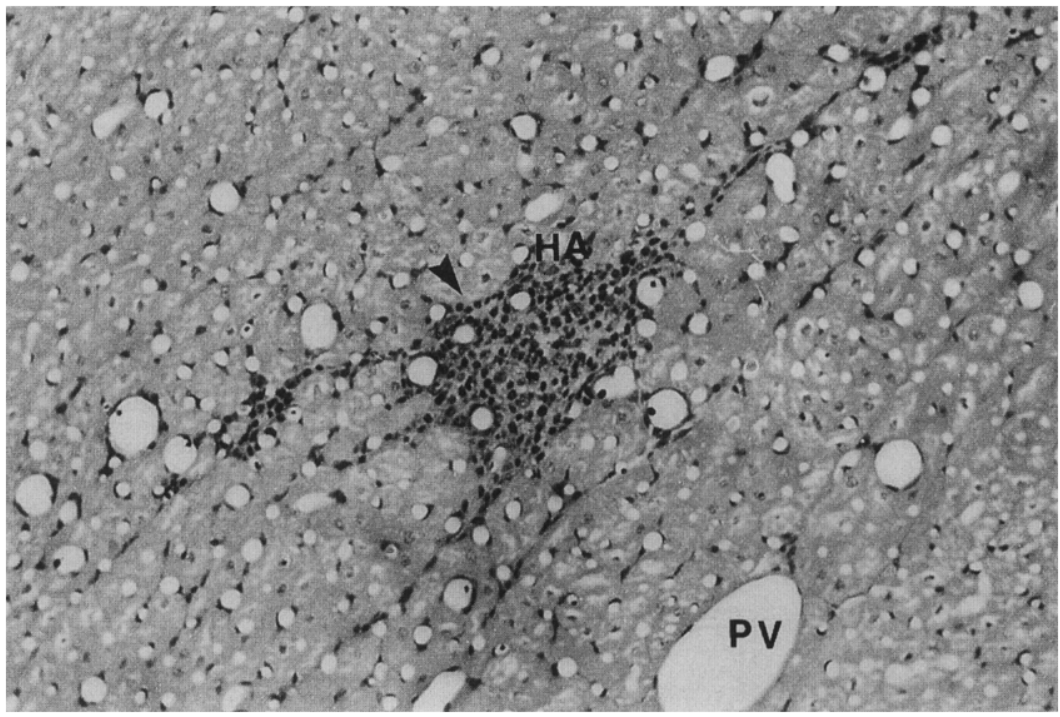

Fig. 1. Injected fetal human atrial cardiomyocytes $(H A)$ are visible as basophilic regions in the left ventricle of the adult pig host $(P V)$. Note the absence of any connective tissue capsule (arrow) and lymphocytes. (Hematoxylin and eosin stain; final magnification $\times 160$.)

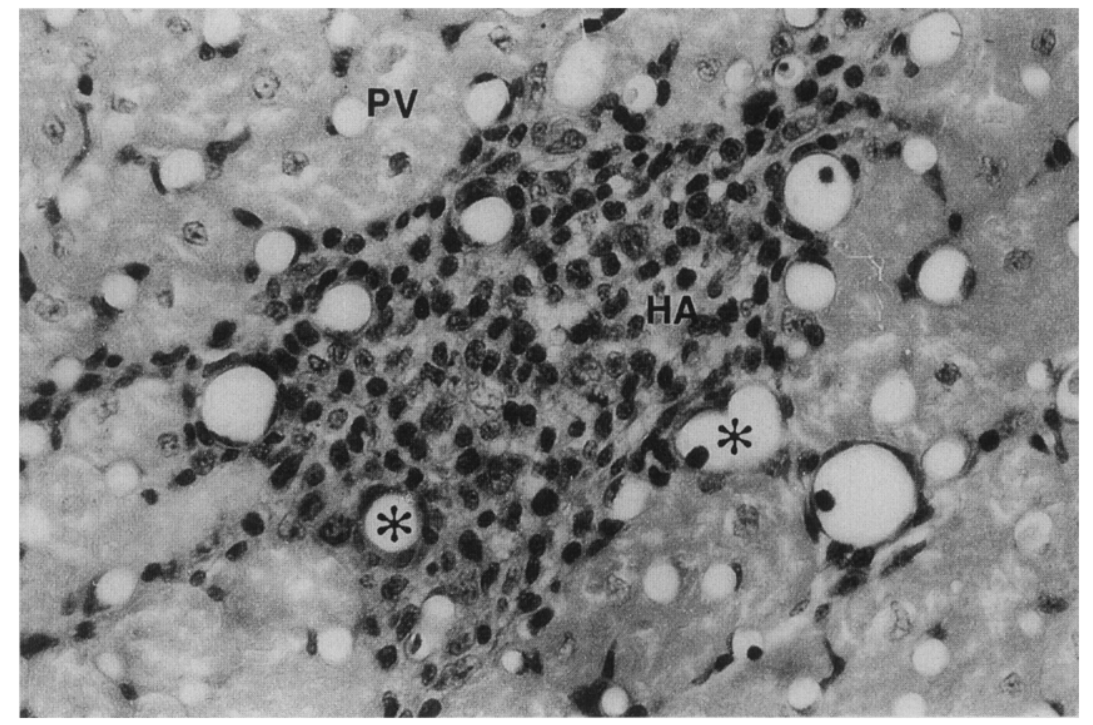

Fig. 2. Injected fetal human atrial cardiomyocytes $(H A)$ also induce what appear to be nascent blood vessels both within the graft and in the interface between graft and host (*). PV, Pig ventricle. Fig. 2 is a higher magnification view of the area demonstrated in Fig. 1. (Hematoxylin and eosin stain; final magnification $\times 400$.)

tion, suggesting that there was no significant myocardial injury from the cellular implantation.

Histologic examination of the excised hearts containing grafted human cardiomyocytes demonstrated no evidence of overt rejection. The cells seemed to align with the native myocytes and most significantly have exerted an angiogenic influence resulting in a marked increase in vascularity in the adjacent host myocardium.

Grafted human atrial cardiomyocytes were easily located by their relatively increased basophilia as compared with the surrounding host ventricle (Fig. 1). Little if any connective tissue was observed surrounding the graft and no lymphocytes or other 


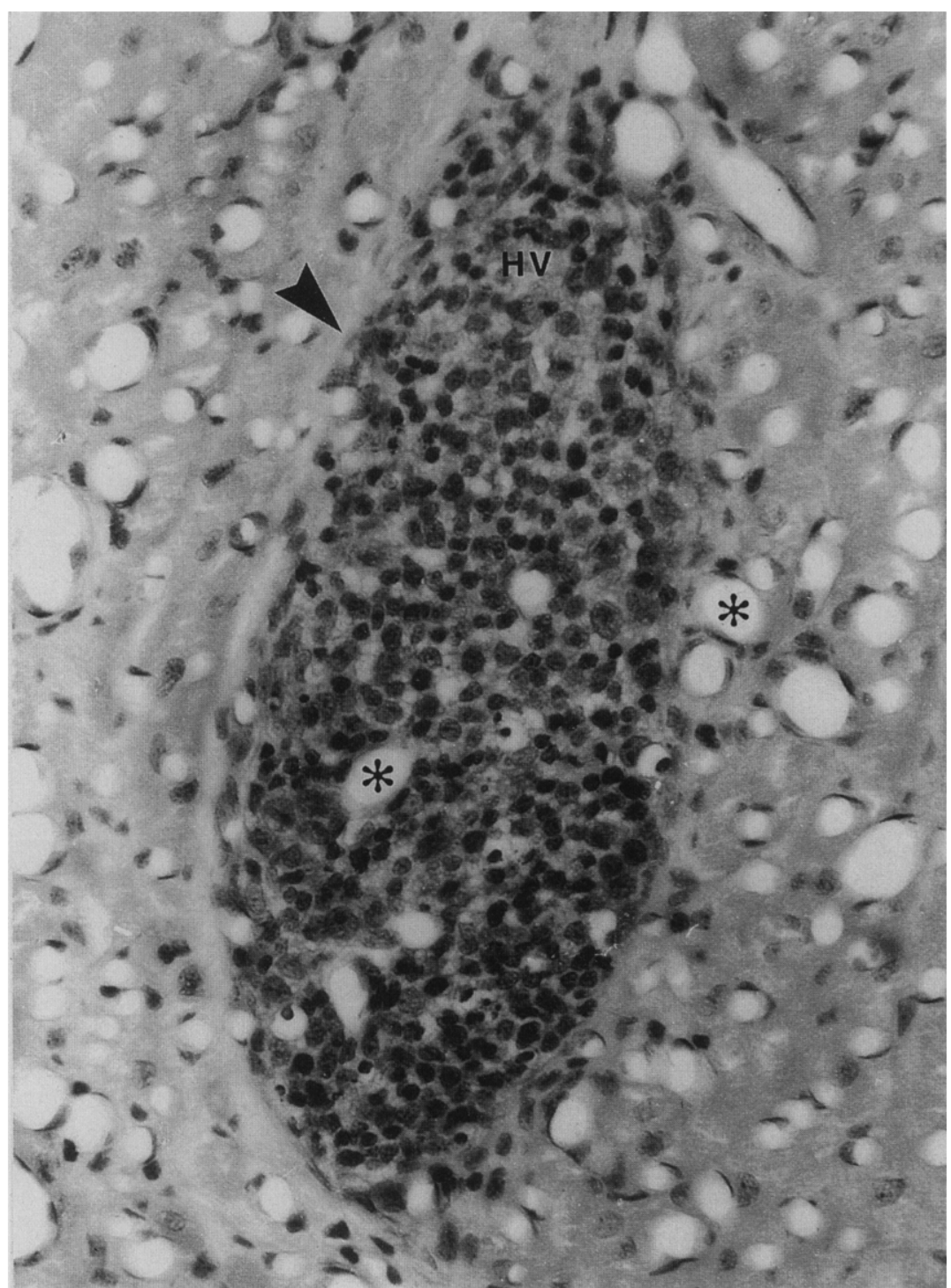

Fig. 3. In addition to fetal human atrial cells, fetal human ventricular cardiomyocytes $(H V)$ also survived transplantation. Transplanted HV cells also induced blood vessels $\left(^{*}\right)$ in the graft area and host ventricle $(*)$. Graft areas lacked any evidence of rejection as shown by the absence of a connective tissue capsule (arrow) and lymphocytes. (Hematoxylin and eosin stain; final magnification $\times 400$.)

immune system cell types were observable with a light microscope. This was clearly evident at higher magnification (Fig. 2). In addition to a lack of encapsulation, blood vessels were observed both within and along the perimeter of the graft (Fig. 2).

Injected human fetal ventricular cardiomyocytes also survived and thrived after intraventricular injection (Fig. 3). At this higher magnification, it is once again clearly evident that there is a lack of encapsulation, an absence of lymphocyte infiltra- tion, and induction of blood vessels both within and surrounding the graft.

AT-1 cells, which were also injected into the ventricles of host pigs, were similar in appearance to the human fetal cardiomyocytes. Using electron microscopy, we have been able to identify nascent junctional contacts between host ventricular cardiomyocytes and transplanted AT-1 cells (Figs. 4 and 5). It is hoped that electron micrographic studies in progress will demonstrate similar junctions in the fetal human studies. 


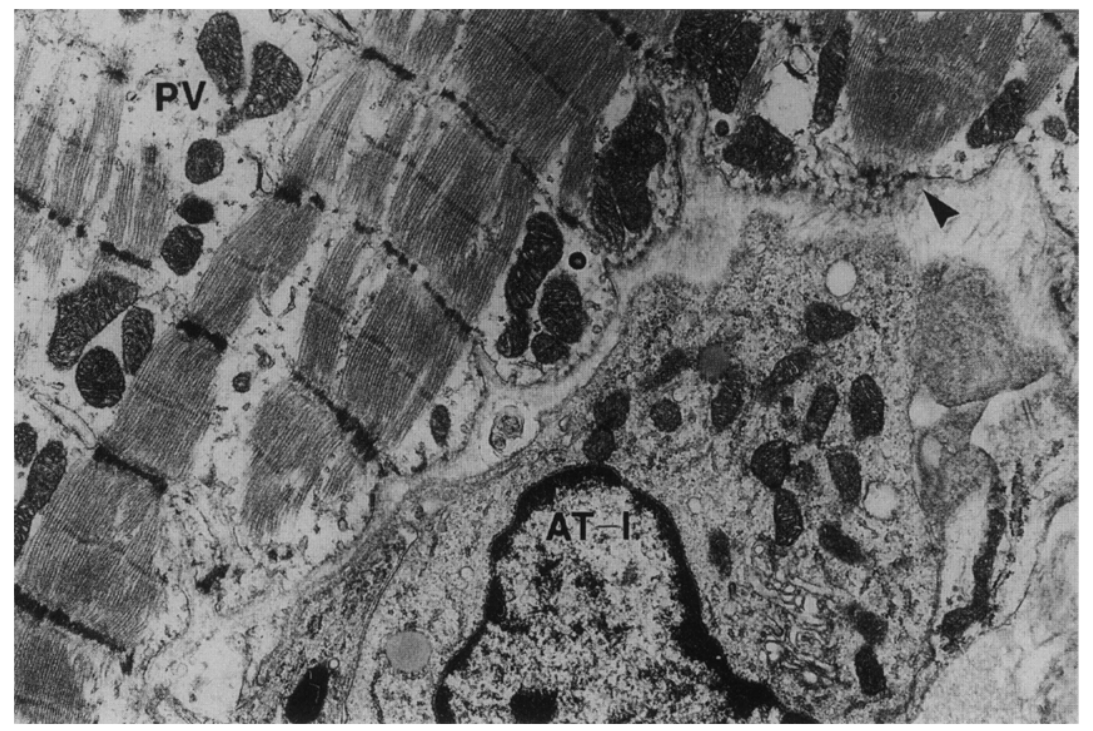

Fig. 4. In studies in which AT-1 cells were injected into host adult pig left ventricles $(P V)$, clear evidence indicated that injected cardiomyocytes could form nascent adherent junctions (arrow) with the host cardiomyocytes. (Transmission electron micrograph; $\times 9000$.)

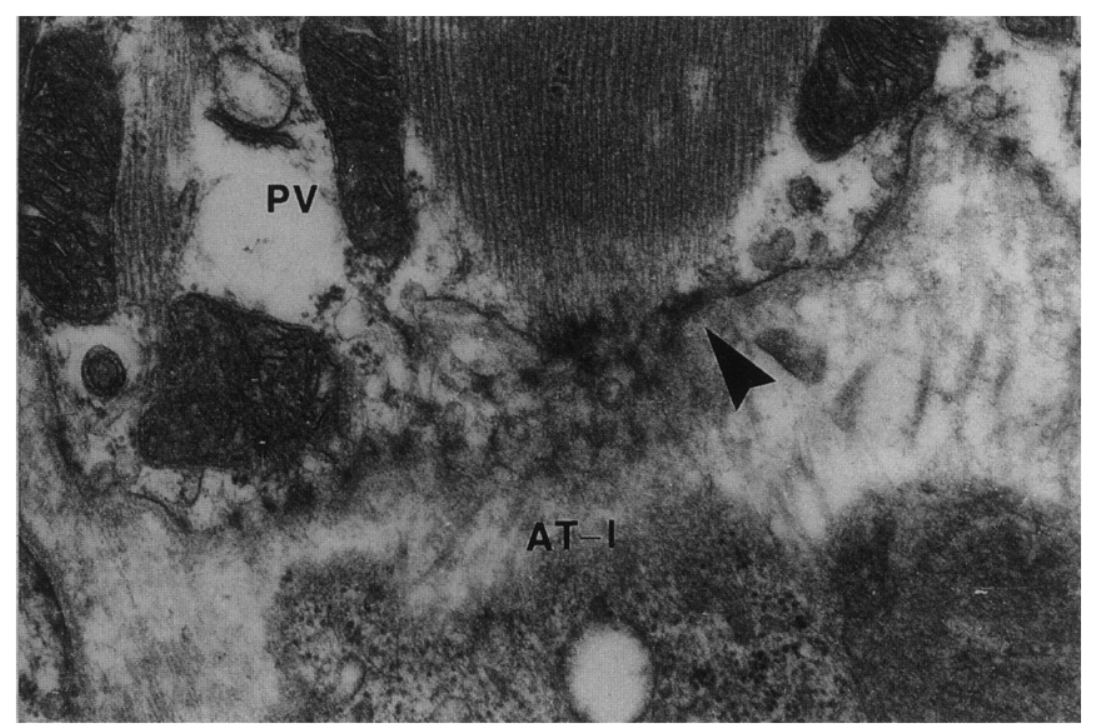

Fig. 5. An enlargement of the area shown in Fig. 4 demonstrating the adherent or nascent intercalated disc (arrow) between an adult pig left ventricular cardiomyocyte $(P V)$ and an injected AT- 1 cell. $(\times 33,300$.)

\section{Discussion}

To understand the obstacles to success in cardiac cellular transplantation, one must first understand the functional characteristics of the mammalian cardiac muscle cell. Unlike skeletal muscles cells, the mammalian cardiac muscle cell cannot regenerate. A full understanding of mechanisms that control the myocyte cell cycle might allow us to design manipulations or procedures that could initiate repair or regeneration of the adult myocardium after injury. This strategy could involve transplantation of pluripotent cardiomyocytes into the intact heart muscle or induction of a reversion of cardiac muscles cells in the intact heart to their biochemical state during early fetal growth when they were actively dividing and proliferating. 
In the rat, at approximately day 17 to 20 of postnatal development, hyperplastic growth ceases for the life of the animal. ${ }^{11}$ From that time on the increase in the muscle mass of the heart is due exclusively to hypertrophy of preexisting cells. It has been estimated that this hypertrophy may be an increase of more than tenfold in volume to form the adult myocyte. The efforts to develop a cell system that can be used to study cardiac muscle cell cycles advanced significantly when it was reported that the large T-antigen of the SV40 virus under control of the atrial natriuretic factor promoter induced large tumors composed of dividing myocytes in the right atria of transgeneic mice. ${ }^{12} \mathrm{~A}$ tumor line was subsequently developed from these mice (AT-1), which was shown to be actively synthesizing deoxyribonucleic acid and dividing in culture. Several days after being plated, these cultured AT-1 tumor cells formed a synchronously contracting monolayer. As demonstrated by cine electron microscopy, as the cells divided they would pull away from adjacent myocytes, cease beating, and progress through mitosis. Subsequently the daughter cells would resume beating shortly after division. ${ }^{13}$ More recently, transgeneic mice have been generated that express the SV40 T-antigen in both atrial and ventricular cardiac myocytes. Myocytes from these transgeneic tumors were able to proliferate in culture while retaining the ability to beat spontaneously. The significance of these collective studies is their demonstration that the differentiated adult cardiac muscle cell from both the ventricle and the atrium can be stimulated to divide without the loss of their highly differentiated state. To further assess the capability to successfully perform cellular transplantation, we undertook our series of experiments in adult swine, using a variety of developed cell lines, fetal swine cardiomyocytes, adult rat atrial and ventricular cardiomyocytes, and finally human fetal myoblasts.

Additionally, the results demonstrate that it is possible to successfully transplant human cardiac muscle cells and myoblasts into immunosuppressed adult animals. Transplanted AT-1 cells and human fetal atrial and ventricular cells may increase the circulation in graft areas, which implies that they may well secrete an angiogenic factor. This increase in microcirculation could provide the grafted cells with a blood supply and also an avenue for removal of cellular debris associated with the primary injury. This angiogenesis is critical to the survival of any grafted cells and may ultimately be responsible for the repair of damaged heart muscle. Future studies will determine whether the use of cells that lack or have reduced immunogenicity, such as the fetal cardiac muscle cells, could greatly improve chances for successful grafting without the use of immunosuppression.

In related studies with tissue culture models, dual labeling experiments demonstrated adherence of transplanted cells to host cells via clearly linked adherence-typed junctions, labeled with anti-pancadherin. Higher magnification with electron microscopy revealed nascent intercalated disks with both a structural component and communicating gap junctions. ${ }^{14}$ Additional experiments are currently underway using transmyocardial ultrasonic microcrystals to measure fractional thickening to determine if the transplanted cells can effect physiologic changes in the host myocardium.

Development of in vivo and in vitro model systems to study direct cardiac muscle cell transplantation into damaged hearts will serve as an experimental tool in evaluating future approaches with homograft and xenograft cardiac muscle cell transplantation used for the direct repair of myocardial damage. Perfection of these cell transplantation systems will also provide an important tool in future cardiac gene therapy paradigms. The morphologic results we have demonstrated, combined with the functional observations that transplanted cells and cardiomyocytes contract in synchrony in co-culture, support the hypothesis that transplanted cardiac muscle cells can form functional couplings in vivo and can potentially repair damaged myocardium.

\section{REFERENCES}

1. Mauro A. Satellite cell of skeletal muscle fibers. J Biophys Biochem Cytol 1961;9:493-7.

2. Claycomb WC. Control of cardiac muscle cell division. Trends Cardiovase Med 1992;2:231-6.

3. Claycomb WC, Palazzo MC. Culturing of the terminally differentiated adult cardiac muscle cell: a light and scanning electron microscope study. Dev Biol 1980;161:249-65.

4. Claycomb WC, Lanson NA Jr. Isolation in culture of terminally differentiated adult cardiac muscle cells. In Vitro 1984;20:647-51.

5. Steinhelper ME, Lanson NA Jr, Delcarpio JB, Wilt AÍ, Claycomb WC, Field LJ. Proliferation in vivo and in culture of differentiated adult atrial cardiomyocytes from transegeneic mice. Am J Physiol 1991;259: H1826-34.

6. Delcarpio JB, Lanson NA Jr, Field LJ, Claycomb WC. Morphological characteristics of cardiomyocytes iso- 
lated from a transplantable cardiac tumor derived from transgeneic mouse (AT-1 cells). Circ Res 1991; 69:1591-600.

7. Claycomb WC, Palazzo MC. Culture of the terminally differentiated cardiac muscle cell: a light and scanning electron microscope study. Dev Biol 1980;80:309-26.

8. Claycomb WC, Bradshaw HD. Acquisition of multiple nuclei and the activity of DNA polymerase alpha and reinitiation of DNA synthesis in terminally differentiated adult cardiac muscle cells in culture. Dev Biol 1983;90:331-7.

9. Claycomb WC, Lanson NA Jr. Isolation and culture of the terminally differentiated adult mammalian cardiac muscle cell. In Vitro 1987;247:701-6.

10. Claycomb WC, Moses RL. Culture of atrial and ventricular cardiac muscle cells from the adult squirrel monkey, Saimiri sciureus. Exp Cell Res 1985;161: 95-100.

11. Claycomb WC. Biochemical aspects of cardiac muscle differentiation, DNA synthesis and nuclear cytoplasmic deoxyribonucleic acid polymerase activity. J Biol Chem 1975;250:3229-35.

12. Field LJ. Atrial natriuretic factor-SV40 T-antigen transgenes produce atrial tumors and cardiac arrhythmias in mice. Science 1988;239:1029-33.

13. Delcarpio JB, Lanson NA Jr, Field IJ, Claycomb WC. Morphological characterization of cardiomyocytes isolated from a transplantable cardiac tumor derived from transgeneic mouse atria (AT1 Cells). Circ Res 1991;69:1591-600.

14. Delcarpio JB, Claycomb WC. Cardiomyocyte transfer into the mammalian heart: cell to cell interactions in vivo and in vitro. Cardiac Growth Regen 1995;752: 267-87.

\section{Discussion}

Dr. Eric A. Rose (New York, N.Y.). At a meeting in which heart volume reduction by excising myocardium or replacing it with inert prosthetic patches has been advo- cated for the surgical treatment of heart failure, I commend Dr. Van Meter and the Ochsner group for courageously pursuing the strategy of trying to improve cardiac function by the actual addition rather than the excision of living contractile tissue to the heart.

The development of the cell lines used in this study required tremendous preliminary effort. This important study proves that the concept of myocardial cell transplantation is feasible in large animals, at least with regard to cell engraftment and the development of a self-sustaining blood supply. As a supplementary observation, we have observed in a comparable rodent model that functional mature gap junctions do develop in the transplanted cells, yet that in the absence of immunosuppression, $85 \%$ of the transplanted cells die by 30 days. Immunosuppression is therefore required for this type of undertaking to be successful at present.

Dr. Van Meter, do you view the propagation of autologous myocardial cells to be feasible ex vivo in the future, or might the strips of myocardium discarded by the reductionists also be a viable source of myocytes for this purpose?

Dr. Van Meter. That is an intriguing question. To be very futuristic and talk more about science fiction perhaps than science, I believe that is feasible. The issue is whether the adult cardiomyocytes can in a sense be "derepressed" by adding the fetal myoblasts that might enable them to differentiate and replicate at the same time.

Our thinking with the myoblasts is that there could be three possible mechanisms of their influence. Probably the least likely is that they actually regenerate in a number to make a significant physiologic difference in the heart's function. The second possibility is that they do exert a change in the milieu of the heart failure muscle and allow those cells to dedifferentiate, replicate, and repair themselves. The final possibility is just that they produce an angiogenic influence. If you could harvest the patient's own cells and somehow influence them in co-culture with the myoblasts and reimplant them, then perhaps you could accomplish regeneration without the problem of immunosuppression. 